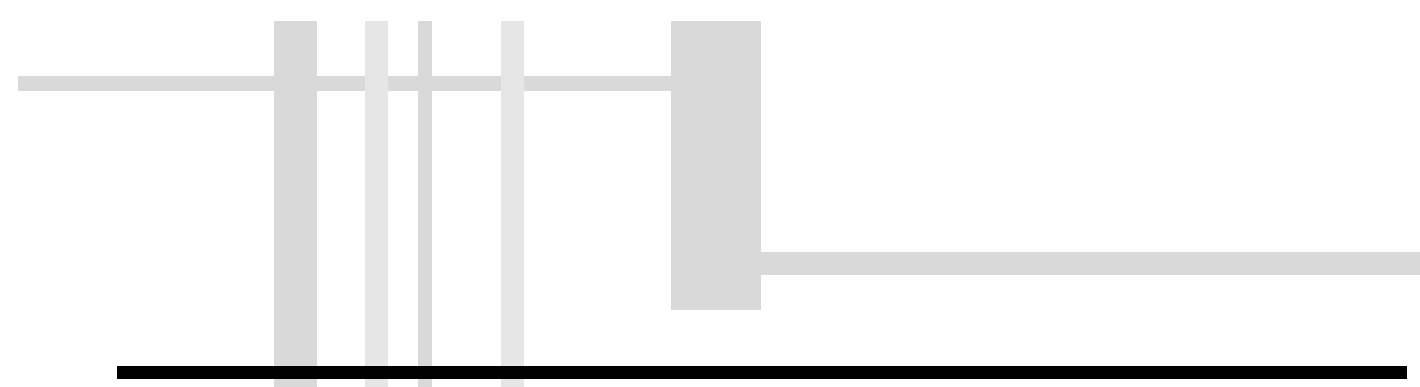

\title{
Propiedades estructurales y electrónicas del Ti calculadas mediante la DFT
}

\section{María Puerto}

alexandrapuerto@gmail.com

William Sosa

woscydm@hotmail.com

Jairo Rodríguez

Grupo Física de Materiales (GFM)

Facultad de Ciencias Básicas

Escuela de Física

Grupo de Estudio de Materiales (GEMA)

Universidad Nacional de Colombia

Departamento de Física

\section{Resumen}

Se han realizado cálculos para determinar las propiedades estructurales y electrónicas del Titanio. En el cálculo teórico se empleó el método de Ondas Planas Aumentadas linealizadas dentro del formalismo de la Teoría del Funcional de la Densidad (DFT) implementado en el código WIEN2k. La energía total del Ti se calculó como una función del volumen y así los datos encontrados se ajustaron a la ecuación de estado de Murnaghan, obteniendo parámetros estructurales tan relevantes como: constantes de red $(a=5,5042$ bohr; $c / a=1,6266)$, la energía de cohesión $\left(E_{0}=-0,8628\right.$ Ry) y el módulo de volumen $\left(\mathrm{B}_{0}=438 \mathrm{GPa}\right)$. Además, se realizó un estudio detallado de las propiedades electrónicas, por medio del cálculo de la densidad de estados y la estructura de bandas de energía.

Palabras clave: titanio, estructura de bandas, DOS, DFT.

\section{Introducción}

El titanio es un metal ligero de color gris plata, abundante en la naturaleza. Posee gran firmeza y dureza, es de baja densidad, presenta alta temperatura de fusión y ductibilidad, 
y muestra muy baja corrosión frente a químicos y al medio ambiente, es un buen conductor del calor y de la electricidad (Zhen, 2000).

Por esto, el interés en su estudio y utilización en el desarrollo de la tecnología aeroespacial, donde el titanio es capaz de soportar las condiciones extremas de frío y calor que se dan en el espacio, al igual que en la industria química, médica y automotriz.

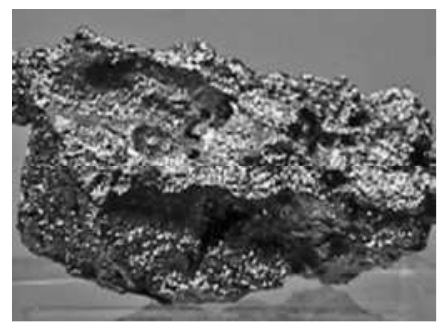

a)

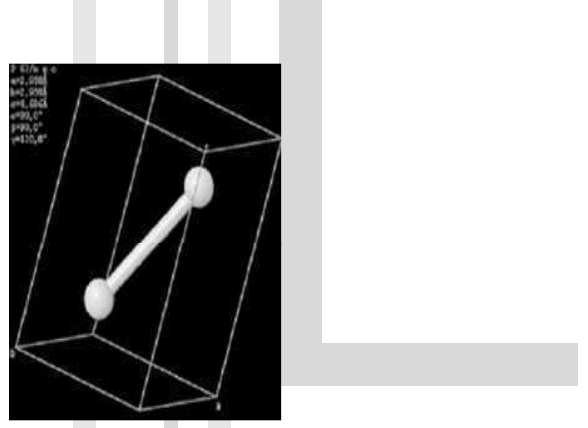

b)

Figura1. a) Titanio en forma natural

b) Modelo de la estructura cristalina

Debido a la gran importancia que tiene este elemento en la actualidad por sus aplicaciones tecnológicas, se pretende calcular algunas de sus propiedades estructurales y electrónicas.

Un sólido está formado por núcleos de átomos localizados y electrones libres dentro de una red periódica. Para analizar las propiedades físicas del sólido es necesario resolver la ecuación de Schrödinger. Esto se convierte en un problema común de muchos cuerpos.

La Teoría del Funcional Densidad (DFT) es un formalismo para tratar el problema de muchos cuerpos a través de aproximaciones. La metodología asociada a la DFT permite introducir los efectos de intercambio y correlación electrónica. Las bases de esta teoría fueron establecidas por Hohenberg-Kohn en 1964 (Hohenberg, 1964) y por Kohn-Sham en 1965 (Kohn, 1965), en ellas se establece un sistema de ecuaciones de uni-partícula no interactuante inmersa en un potencial efectivo para solucionar el problema de muchas partículas interactuantes.

La estrategia de la DFT es escribir las propiedades de un sistema mediante funcionales de la densidad. Para definirlo aparecen propuestas iniciales como la Aproximación de la Densidad Local (LDA) en donde la idea central para definir el funcional es la hipótesis de un gas uniforme de electrones; y la Aproximación de Gradiente Generalizado (GGA), una aproximación más sofisticada que incorpora el gradiente de esta densidad. Por otro lado, la DFT es un método de potencial completo, el cual usa como funciones solución ondas planas aumentadas y linealizadas FP-LAPW y el método de orbitales locales. 
Mediante DFT es posible calcular la energía total del sistema para luego ajustarla a una ecuación de estado conocida como ecuación de estado de Murnaghan, que viene dada por la expresión:

$$
E(V)=E\left(V_{0}\right)+\left(\frac{B_{0} V_{0}}{B_{0}^{\prime}}\right)+\left[\frac{\left(V_{0} / V\right)^{B^{\prime} 0}}{B_{0}^{\prime}-1}+1\right]-\left(\frac{B_{0} V_{0}}{B_{0}^{\prime}-1}\right)
$$

Donde $V$ es el volumen de la celda primitiva, $V_{0}$ es el volumen en equilibrio, $B_{0}$ es el módulo de volumen y $\mathrm{B}_{0}$ es la derivada del módulo de volumen respecto a la presión calculada en $\mathrm{V}_{0}$.

\section{Detalles del cálculo}

El titanio presenta una estructura cristalina hexagonal del tipo $P 6(3) / \mathrm{mmc}$, cuyas posiciones atómicas se muestran en la tabla 1.

Tabla 1. Posiciones atómicas para el titanio

\begin{tabular}{lccc}
\hline Átomo & $\mathbf{x}$ & $\mathbf{y}$ & $\mathbf{z}$ \\
\hline $\mathrm{Ti}$ & $1 / 3$ & $2 / 3$ & $1 / 4$ \\
\hline
\end{tabular}

En el cálculo se implementó el programa Wien 2K (Blaha, 2001) utilizando la aproximación de gradiente generalizado (GGA) con la parametrización Pedew-BurkeErnzerhof (Perdew. 1996) para tratar los efectos de correlación e intercambio. Se tomó un valor de energía para separar los estados del core y valencia de -6,0 Ry. Como criterio de convergencia para la energía se usó un valor de 0,0001 Ry. Se utilizó un $G_{\max }=12 R^{1 / 2}, G_{\min }=8,42 R^{1 / 2} y_{L_{\text {max }}}=10$. Se usaron 5000 vectores $\mathrm{K}$ en la primera zona de Brillouin. Los valores de energía se ajustaron mediante la ecuación de estado de Murnaghan. Adicionalmente, se realizó un cálculo de estructura de bandas de energía y la densidad de estados en el volumen de equilibrio.

\section{Resultados y discusión}

El ajuste de la energía vs volumen, a partir de la ecuación de estado de Murnaghan, permitió calcular los valores para los parámetros estructurales del Ti, que se muestran en la tabla 2.

La figura 2 muestra el ajuste a la ecuación de estado de Murnaghan, para la energía en función del volumen. 
Tabla 2. Parámetros estructurales correspondientes al ajuste de la ecuación de estado de Murnagahan para el Ti

\begin{tabular}{|c|c|c|c|c|}
\hline \multirow[b]{2}{*}{$\begin{array}{c}\text { Constante de } \\
\text { red a [a.u.] }\end{array}$} & \multicolumn{3}{|c|}{ Parámetros calculados } & \multirow[b]{2}{*}{$\begin{array}{c}\text { Energía de } \\
\text { cohesión [Ry] }\end{array}$} \\
\hline & $\mathrm{C} / \mathrm{a}$ & $\begin{array}{c}\text { Volumen } \\
\text { atómico [a.U3] }\end{array}$ & $\begin{array}{c}\text { Módulo de } \\
\text { volumen [GPa] }\end{array}$ & \\
\hline 5,5042 & 1,6266 & 234,9078 & 438,0000 & $-0,8628$ \\
\hline
\end{tabular}

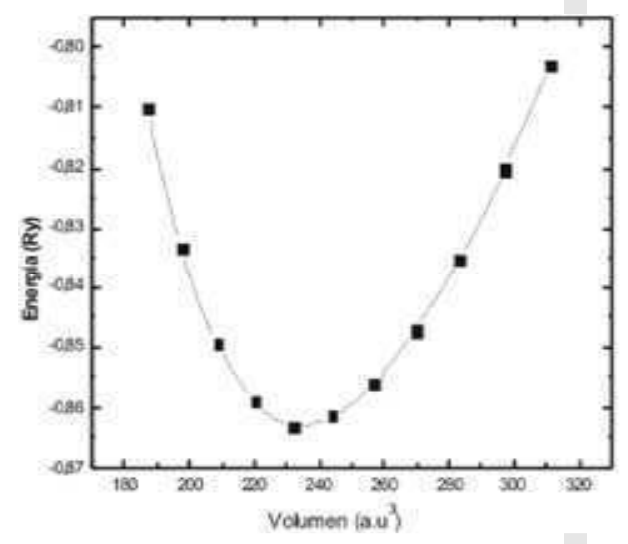

Figura 2. Energía total en función del volumen de la celda para el Ti

En la figura 2 se indica el valor para la energía de cohesión y el valor para el volumen que minimiza esta energía. El valor de la energía mínima verifica la estabilidad de la estructura del Ti y el valor del volumen indica el valor del volumen de la celda primitiva del cristal.

En lo referente a las características electrónicas, las bandas de energía y la densidad de estados se muestran en la figura 3. Aquí se observa la energía en función del vector de onda $k$ para algunas direcciones de alta simetría. La escala de energía cero corresponde a la energía del nivel de Fermi que dio un valor de $\mathrm{E}_{\mathrm{F}}=0,56738 \mathrm{Ry}$.

En esta figura se pueden identificar dos regiones principales: de $-9 \mathrm{eV}$ a $0 \mathrm{eV}$ la región de valencia y la región de la banda de conducción, ubicada sobre el nivel de Fermi. De esta manera se evidencia el carácter conductor del Ti, debido a la concentración de un gran número de estados alrededor del nivel de Fermi (no existe ningún gap de energía), las cuales también pueden asociarse con la densidad de estados alrededor del mismo valor de energía de Fermi. 


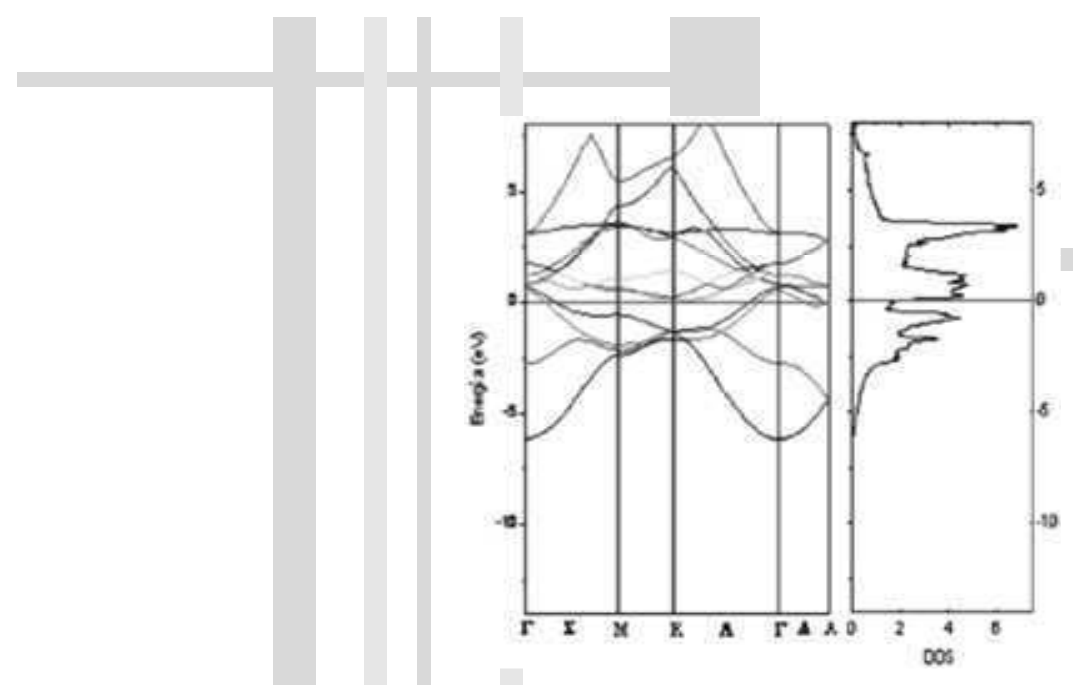

Figura 3. Estructura de bandas de energía y densidad de estados para el Ti

\section{Conclusiones}

A través de la DFT y el método FP-LAPW se calcularon las propiedades estructurales del Ti en su fase $P$ 6(3)/mmc, obteniéndose un parámetro de red óptimo con el cual se determinó la densidad de estados y la estructura de bandas de energía. Estas permiten evidenciar el carácter conductor de este elemento. También se demostró el carácter estable de esta estructura, debido a que se obtuvo un valor negativo para la energía de cohesión $\mathrm{E}_{0}=-0,86 \mathrm{Ry}$.

\section{Referencias bibliográficas}

Blaha, P., Schwarz, K., Madsen, G., Kvasnicka, D., y Luitz, J. (2001). An Augmented Plane Wave Plus Local Orbitals Program for Calculating Crystal Properties. Austria: Vienna University of Technology.

Hohenberg, P., y Kohn, W. (1964). Inhomogeneous Electron Gas. Physical Review (Vol. 136).

Kohn, W., y Scham, L. (S.F.). Self- Consistent Equations Including Exchange and Correlation Effects. Physical Review (Vol. 140).

Perdew, J., Burke, K., y Ernzerhof, M. (1996). Generalized Gradient Approximation Made Simple. Physical Review (Vol. 77).

Zhen, L., y Run Ze, L. (2000). Non-aerospace application of Ti materials with a great many social and economic benefits in China. En: ScienceDirect Database. 